\title{
The Quality of Data Center Infrastructure Management (DCIM)
}

\author{
Velpula Sundara Ratnam, K.S Sarma
}

\begin{abstract}
Now-a-days, Data Center Infrastructure Management is increasing workplace efficiency, reducing overhead costs, and maintaining availability are some of the top priorities for data center administrators. The markets of data center have evolved not only in reply to the quantity of data, but also to increasing demands approximately connectivity up-time, security, and lesser IT costs. Information Technology is growing more rapidly than ever and new challenges appear every day. Various challenges are data storage and processing, and data centers have a critical role in this situation. As data centers get bigger and bigger, more energy is required and availability is primary. In this paper it is discussed the quality of DCIM by taking into requirements and the quality evaluation problem, here is a proposed method to analyze the quality of DCIM for energy costs, availability and new systems of data center infrastructure management (DCIM). With the proposed method of evolution will definitely resolve the problems of Data Centers so that the throughput of data centre will increase efficiently.
\end{abstract}

Keywords : About four key words or phrases in alphabetical order, separated by commas.

\section{INTRODUCTION}

Data center infrastructure management (DCIM) is the junction of I.T and building services functions within an organization. The objective of a DCIM idea is to provide administrators with a holistic and actual view of a data center's performance so that energy, equipments and floor space are used as powerfully as potential.At present data centers are more and more complex, more interdependent and more vital than ever before. Thisscenario has led to the need for more intellectual and computerized IT infrastructure management. The tools which allow the data center team to successfully and competently operate this multifaceted environment have been grouped into a classification of solutions known jointly as Data Center Infrastructure Management (DCIM). Gartner defines Data Center Infrastructure Management as "tools that examine, measure, deal with and/or control data center use and energy utilization of all IT related equipment like servers, storage and network switches, and amenities infrastructure components such as power distribution units and computer room air conditioners." Multiple Data Center Infrastructure Management models have been put forward by analyst firms such as Gartner, Forrester and the Group. In many respects,

Revised Manuscript Received on 14 August, 2019.

* Correspondence Author

Velpula Sundara Ratnam,

K.S Sarma , there are slight differences between the different views ofDCIM.In earlier days the concept of a data center was different that of today. In the history there was not a really devoted place to host servers, switches and all devices that run the computer network, and these equipments were located in common adapted rooms as possible. From the increase of internet and all connected services coming in current years like Cloud Computing, Virtualization, Parallel Programming, exigencies regarding up-time and service level agreements are in all business contracts. In addition to this, new data centers will represent an increase of about $50 \%$ more electrical energy than the year before. This dramatic energy situation is pushing several new researches that look for economy, management and control. As Data center infrastructure management are extremely new platforms with varied industrial solutions and diverse characteristics, and considering their importance for the entire information technology. A suggestion for a new Evaluation Method is presented and sample results are showed.

\section{DCIMCONCEPTS}

The main objective of DCIM is to allow managers to get real time information from the data center environment, though the definition of DCIM hasn't changed much over the years, the model has changed considerably. Once accepted and considered a set of different disparate tools, expectations of DCIM have evolved from tools to a platform. A proper platform is required because integration capabilities are no longer considered elective. Neither single technology solution nor vendor is realistic for data center evolution. By means of information at hands, managers will be able to perform actions directly on the infrastructure components, simulate work, analyze and plan changes when appropriate. In a extremely competitive situation, operators of data centers are increasingly looking for new operational efficiencies as the management of data centers, their IT systems and services. Separate actions increasingly converge into more holistic operation.

Data center infrastructure management can be understoodat the same time as a technicaland as a business tool. It is a efficient technical tool for the reason that it permits monitoring and analysis of physical infrastructure, and also interfering on it by actuators. Applying a DCIM podium on the data center, resources management is optimized, devices performance is drastically improved, energy is saved, operational costs are reduced and next steps of growing are previewed. It is a business tool since it is oriented

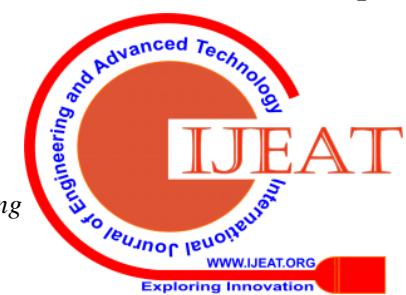


to cost reduction, management and general performance enhancement.

DCIM may be a junction of a number of different platforms integrated, or just a single platform developed for this proposal. Generally speaking it combines different technologies for:

\author{
Infrastructure environment monitoring. \\ Pathways and area management. \\ Operational work planning. \\ Energy efficiency. \\ General performance optimization. \\ Physical security.
}

DCIM platforms are developing rapidly from just a few years and are a new branching of automation systems. Its market is new, strong and high specialized. Presently, it is not a leader product and no standards yet. Approximately just $1 \%$ of penetrations in data centers in 2012, DCIM systems are expected to be present in approximately $60 \%$ of all data centers in 2015.

\section{DCIMOBJECTIVES}

Three keywords to define DCIM objectives and why they are so necessary: availability, manageability and economy.Availability and accessibility means that the data center is working appropriately every time when any service is demanded from it. But every now and then it may not occur, and all efforts are done to get so much uptime as possible. The Uptime Institute normally defines standards, to orient technical solutions and acceptable levels of downtime. Data center infrastructure management (DCIM) systems will help on data center availability considering, for instance, the impact of new devices installation and if the power circuits will support them, or if there is sufficient space within the cabinets. Different other questions to consider are as: which are the cabinets that have electrical circuits with enough load, and how to connect equipments on it? Any circuit is likely to use or is there some kind of balancing to take care? Is there any option to have a "crash" if one new device is connected? Where are the vital points of fail on heat generation and how air conditioningsystems are working on them? Are there electrical panels congested or with some kind of risk? Are there alternating or minor fails, not clearly perceivable, that could suddenly interrupt the data center availability?

Manageability means the ability of managers to administrate the complex data center operations and maintenance. To continue the data center available, all its different subsystems are working in parallel and are interdependent, and this is an extra difficulty because managers must look on, for instance, so different aspects such as staff, energy, costs and security. DCIM systems will efficiently help management by organizing staff roles and responsibilities, controlling authorization accesses and/or actuation, creating forms and reports, showing plots online about all data collected from the field, and several other different information that are important to have an in deep view of the data center. Data center infrastructure management (DCIM) will help managers on creating and sending service orders (for maintenance or new installations), defining where are the best ways and places to locate new devices or relocate existing ones, distributing logins and passwords for staff or visitors access, doing continuous verifications of PUE - Power Usage Effectiveness which defines the energy effectiveness of the data center, establishing maintenance routines and getting response from them, besides several other regular management processes that are usual or specific for each datacenter.

Economy is the third important keyword and defines how to reduce continuously the cost of data center operation and growth. The Power Usage Effectiveness (PUE) cited before has become one of the important main index numbers to show how the data center general energy efficiency is. DCIM systems can work out electrical power utilization of all circuits and then calculate the rate between general data center electrical power consumption and I.T (information technology) devices consumption.

\section{DCIMFUNCTIONS}

Data center infrastructure management investments are reasonable, and that means seeking out an appropriate solution with a key set of fundamental features, namely the following:

1. Real-time visualizations

Researchers will talk a lot about the (IoT) Internet of Things, and the deployment of sensors that can collect data in the data center in real time and with extraordinary accuracy. The next second half of that process involves subsequently analyzing that data as quickly as it is collected to monitor a facility's health in real time in order to continuously facilitate the following:

a. Health: At any given instant, is everything operating to specifications?

b. Performance: How consistent is the facility right now?

c. Efficiency: Are the resources really contributing to the health and performance of the facility?

In order to answer the above important questions, DCIM compiles data collected through real-time monitoring, including rack temperature, humidity, airflow, power consumption and IT load, and visualizes that real-time information in a single, coherent interface. If something is wrong, it is not possible just know through your alerts - you'll also see it. This facility overview is perhaps the most powerful function that modern DCIM has to offer. A universal view of your data center can improve reliability, performance and efficiency.

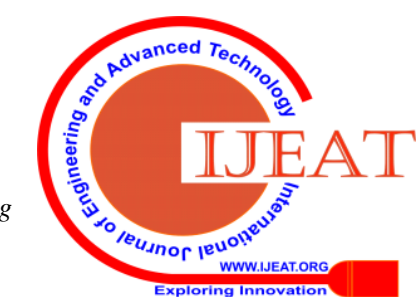




\section{Asset and change management}

According to Data Center Knowledge Contributor Michael Potts, 26 percent of survey respondents said that they would be able to find a specific server within minutes of being asked about it. In the meantime, 58 percent said that they could find it in four hours, and 20 percent said that it would take them a day or more to locate that equipment.

The purpose of asset management is to generate a virtual inventory of all facility equipment, including servers, cooling equipment, network switches, power distribution units (PDUs), facility monitors and so on. The other side, asset management provides an operational status for that piece of equipment and shows what other equipment it may be connected to or influencing at a given moment.

Finally, because the objective is to keep this information accurate up to the second, asset management facilitates, change management, which is the documentation of any changes that are made to any assets in this effective index of the data center.

\section{Capacity planning}

The majority optimized data centers can get blindsided by growth if they do not adequately account for changes in resource consumption over a period of time. This goes beyond recording events. It requires a replete understanding of a facility's resource limitations. This includes footstep (physical space) and IT load, but also cooling capacity and power utilization.

This is where DCIM's planning capabilities come into the picture. By tracking resource utilization trends over time and analyzing them, facility managers can follow growth patterns to understand resource capacities and adjust according to future requirements. A DCIM solution will also mark predictive modeling that allows for the simulation of hypothetical facility setups and scenarios.

Although it appears third in this list, capacity planning is no less important than anything preceding it, especially taking into consideration the rapid rate at which data is being created, physical activities are being digitized and workflow functions are being automated. Change is the only constant now. It helps you attain these goals with functionality for effectively managing your inventory, cutting power utilization, eliminating downtime and optimizing data center operations. But you don't immediately need every DCIM capability. Deciding which capabilities to implement depends on your priorities. Start with issues that most negatively impact operations. Are you spending money on equipment you don't need? DCIM inventory tools provide an accurate, real-time inventory of every asset's floor or rack position with modeling to show you the impact of change. Are you experiencing too much downtime? DCIM's insight into real-time power consumption and dependencies between devices helps you reduce energy usage and unplanned out

\section{DCIM IN MODERN DATA CENTERS}

Some of the capabilities offered by DCIM solutions on this front cost savings through energy management The greatest operational costs of running a typical data center is the cost of the power required to operate the high-performance servers and air-conditioning hardware that it houses. The DCIM's amalgamation with the building's power feeds and ability to monitor IT and non-IT loads allows it to easily track the PUE, or power usage effectiveness, within the data center, keeping it in a unique position to facilityefficientenergyuse. The cost savings of more efficient energy usage to one side, organizations with corporate energy efficiency initiatives can also look to DCIM to better manage and optimize energy use across their complete infrastructure, and do their part to help the environment.

Monitoring for increased reliability

At the fundamental level, the DCIM works in the background to collect comprehensive log entries from the hundreds or even thousands of individual devices and components within the data center, including parameters such as energy utilization, temperature, humidity and airflow. The resulting archive of records facilitates simple analysis, and is also helpful in the creation of a virtual model of the infrastructure to digitally map the relationships between all these components. This model also lends itself to proactive and easy maintenance such as using an analytical engine to scour for inefficiencies or weak links within the environment, and can make difference when performing a root cause investigation in the event of an outage or failure.

Better

capacity

planning

The effectiveness of a data center is highly dependent on appropriate capacity planning, and entails detailed load calculations under normal and peak load conditions. Facility managers normally rely on capacity planning tools to determine the resources and power draw that a data center must support.

The information such as existing resource use and cooling patterns that a DCIM can provide would be a shot in the arm for standalone planning tools. In addition, being able to accurately predict when a resource will be exhausted can also ensure that all new builds or expansion plans are directly aligned with actual demand.

Taking into account, the requirements and the quality evaluation problem here is proposed a method to analyze the DCIM quality development. The analysis considers fivecriteria:

DataCriterion,

Automation Criterion,

DiagnosisCriterion,

InterfaceCriterion,

ManagementCriterion. 
The criteria cover up different aspects of all areas that DCIM platform must support. The Data Criterion is associated to the collecting and storage of information from the data center environment as a whole. The Automation standard considers how DCIM interacts with all datacenter sub-systems in terms of automatic controls, and also how it dialogs with legacy software, if existent. Diagnosis Criterion considers the capability of DCIM to analyze and create real time diagnostics. Interface Criterion clearly defines the way that DCIM can show to users the information from data center, diagnostic results and tools for actuation (buttons, forms, etc). Management Criterion considers DCIM capabilities associated to planning, simulation and intelligence, helpingmanagers on day to day and future management of the data center.

The estimate of each criterion is defined by a table filled with several topics that constitute its specific features. For each topic the model projected considers three levels of Quality, as shown at TableII.

TABLE II LEVELS OF QUALITY

\begin{tabular}{|l|l|}
\hline Level (ri) & Description \\
\hline 0 & $\begin{array}{l}\text { Feature does not gather the } \\
\text { requirement }\end{array}$ \\
\hline 1 & $\begin{array}{l}\text { Feature partly meets the } \\
\text { condition }\end{array}$ \\
\hline 2 & Feature meets the requirement \\
\hline
\end{tabular}

Each DCIM criterion development Quality is then defined by the mean of all level topics, according to equation (1), where $\mathrm{n}$ is the number of topics of the Criterion, liis the weight of the requirement, and riis the level of its Quality.

$$
\lceil\bar{m}\rceil=\left\lceil\frac{\sum_{i=1}^{n}\left(l_{i} * r_{i}\right)}{\sum_{i=1}^{n} l_{i}}\right\rceil(1)
$$

However, each Criterion has requirements for each feature, which are detailed on specific forms, as shown below (Tables III to VII). It is significant to notice that these forms are a proposal and do not exhaust all possibilities. Different new requirements and features can be defined and then improve the forms and evaluation results.

\section{Data Selection}

The focal point of the Criterion is the evaluation of how DCIM is collecting and storing information from data center environment. And also, it analyzes the qualitative, quantitative scope of these data. Table III explains the evaluation requirements shared on different topics.

\section{Automation Selection}

Automation principle considers the automatic systems associated to the several subsystems present in a data center, like cooling, security, power and so on. It defines requirements for manage and interaction between the subsystems, and also with legacy software, if existent. Table
IV presents the automation criterion evaluation requirements and different topics.

\section{DiagnosisSelection}

The main purpose of this Criterion is the evaluation of DCIM analysis capabilities, considering, for instance, analysis of points of risk or fail, critical areas within the data center, and how to manage them. Table $\mathrm{V}$ shows the diagnosiscriterion estimation requirements and topics.

\section{TABLE IIIDATA CRITERION EVALUATION REQUIREMENTS}

\begin{tabular}{|c|c|c|c|c|}
\hline & & 0 & 1 & 2 \\
\hline 1 & Environmental Data Monitoring & & & \\
\hline 1.1 & DCIM collects, stores power data & & & \\
\hline 1.2 & $\begin{array}{l}\text { DCIM collects and stores humidity } \\
\text { data }\end{array}$ & & & \\
\hline 1.3 & $\begin{array}{l}\text { DCIM collects } \quad \& \quad \text { stores } \\
\text { temperature data }\end{array}$ & & & \\
\hline & $\begin{array}{l}\text { DCIM detects smoke and stores } \\
\text { data }\end{array}$ & & & \\
\hline 1.5 & $\begin{array}{l}\text { DCIM detects fluid flow and stores } \\
\text { data }\end{array}$ & & & \\
\hline 1.6 & $\begin{array}{l}\text { DCIM detects leakage and stores } \\
\text { data }\end{array}$ & & & \\
\hline 1.7 & $\begin{array}{l}\text { DCIM collects \& stores CCTV } \\
\text { Images }\end{array}$ & & & \\
\hline & $\begin{array}{l}\text { DCIM registers and stores } \\
\text { information from datacenter } \\
\text { devices and components }\end{array}$ & & & \\
\hline 1.9 & $\begin{array}{l}\text { DCIM collects \& stores Access } \\
\text { control data }\end{array}$ & & & \\
\hline 2 & Monitoring Scope & & & \\
\hline 2.1 & $\begin{array}{l}\text { DCIM monitors each Rack/cabinet } \\
\text { unit }\end{array}$ & & & \\
\hline 2.2 & DCIM monitors each Room & & & \\
\hline 2.3 & Real time monitoring (online) & & & \\
\hline 3 & Interval between data collects & & & \\
\hline 3.1 & Fraction of seconds & & & \\
\hline 3.2 & Fraction of minutes & & & \\
\hline 3.3 & Fraction of hours & & & \\
\hline
\end{tabular}

TABLE IV AUTOMATION CRITERION EVALUATION REQUIREMENTS

\begin{tabular}{|l|l|l|l|l|}
\hline 1 & Subsystems integration & 0 & 1 & 2 \\
\hline & $\begin{array}{l}\text { Mechanization is integrated } \\
\text { to data monitoring,alerting } \\
\text { tools, security access control } \\
\text { \& CCTV. }\end{array}$ & & & \\
\hline 1.2 & $\begin{array}{l}\text { Access control such as } \\
\text { rooms and cabinets and } \\
\text { CCTVare integrated. }\end{array}$ & & & \\
\hline 2 & Heterogeneous platforms. & & & \\
\hline
\end{tabular}




\begin{tabular}{|c|c|}
\hline 2.1 & $\begin{array}{l}\text { Heterogeneous } \mathrm{H} / \mathrm{W} \\
\text { platforms are integratedin a } \\
\text { distinctive console }\end{array}$ \\
\hline 2.2 & $\begin{array}{l}\text { H/Wusing different open } \\
\text { protocols such as Modbus, } \\
\text { BACnet, CAN, SMNP, can } \\
\text { beintegrated. }\end{array}$ \\
\hline 3 & Limits \\
\hline 3.1 & $\begin{array}{l}\text { Limits can be set for } \\
\text { predictive alarms/warning. }\end{array}$ \\
\hline 3.2 & $\begin{array}{l}\text { Different limits for diverse } \\
\text { devices can be set for } \\
\text { predictive alarms/warning } \\
\text { on thesimilarplatform. }\end{array}$ \\
\hline 4 & Control \\
\hline 4.1 & $\begin{array}{l}\text { In one control network all } \\
\text { sub-systems areintegrated. }\end{array}$ \\
\hline 5 & Operation \\
\hline 5.1 & $\begin{array}{l}\text { DCIM operates contingency } \\
\text { equipment in case } \\
\text { ofpredictive alarming. }\end{array}$ \\
\hline 5.2 & $\begin{array}{l}\text { DCIM controls\& operates } \\
\text { various } \\
\text { heterogeneousdevices, even } \\
\text { if they belong to different } \\
\text { vendors. }\end{array}$ \\
\hline
\end{tabular}

\section{InterfaceSelection}

This principle is focused on the presentation of all information and diagnosis operated by the DCIM platformand it analyses how this information is showed and formatted, and also the presentation of outcome and tools for actuation (buttons, forms, etc). Table VI shows the interface criterion assessment requirements andtopics.

\section{ManagementCriterion}

This criterion analyses and focuses the DCIM Quality to deal with planning, simulation and intelligence tools, aiming the management of day to day and future of the data center. Basically related to all other criteria, it may coordinate the evolution and growth of the data center itself. Table VII shows the management principle evaluation requirements andtopics.

Considering the five criteria, any DCIM platform can be analyzed. Each one of the criteria has a final value and calculated by the equation (1). The Comparisons between different products can be done criterion by criterion, depending on what is important for a specific use or application.

The ultimate Quality evaluation for a DCIM platform will be given by the sum of each one of the values obtained. Equation (2) shows the calculus for a DCIM Quality generalstage.

So how does a vendorassess and organize a DCIM solution for their data center? Unlike in the early days where only a handful of DCIM offerings were available, there are now scores of vendors offering a diverse range of solutions. The disadvantage would be how it is no longer practical to explain all of them individually, given the intricacy and sheer amount of time needed for a proper evaluation.

Instead of trying to find out the "perfect" solution, organizations should move toward the search for an ideal DCIM solution with their end objectives firmly in mind. In this scenario, this includes factors such as performance in operating environments, support of existing hardware, and level of integration with existing IT management software.

In this situation, it makes sense to position an ecosystem that is explicitly supported by the DCIM from the get-go, such as PDU (Power Distribution Units), STS (Static Transfer Switch) and ancillary equipment together with access and surveillance hardware. Delta with its collection of integrated components can hence serve as a one-stop-shop for the data center requirements of facility managers.

Where is DCIM headed though, and what can we anticipate from it? One side, the current data center is already geared for seamless 24-by-7 operation and business continuity, a modern DCIM solution can enable an even higher level of automation to forecast and resolve problems before they occur.

In fact, expect a new generation of data centers to be installed with pervasive Internet of Things appliances and sensors, coupled with deep software integration to the compute, storage and network stacks. Different advanced machine learning algorithms would continuously fit in a thousand different parameters to ensure that power and cooling precisely mirror the ebb and flow of compute workloads.

\section{RESULTS APPLYING THEMETHOD}

Applying, first of all, the Data Criterion (Table III) and taking into account the weight liequal to one, the results are 2.00 and 1.60 for DataFaz and DCIM2 respectively. The outcomes for Automation Criterion are 1.89 and 1.50 respectively. The results for Diagnosis Criterion are 1.70 and 2.00. The results for Interface Criterion are 2.00 and 0.90 . The results for Management Criterion are 1.38 and 1.76. Theoutcome show that DataFaz has animproved level of Quality then DCIM2 at Data, Interface and Automation Criteria, whereas DCIM2 is better at Management and Diagnosis Criteria. Considering the general value of Quality, according to equation (2), DataFaz has a Quality level 8.96, and DCIM2 has Quality level 7.76. Figure 1 represents a chart with both results plotted on five axes, where DataFaz is represented by the continuous line and DCIM2 by the dashed line.

The comparison here is comparative and only for demonstrative reasons, expecting to illustrate the modelcapabilities. The model proposed necessarily must havemore detailed requirements in each of the criteria, and 
developers from academic and commercial background can help enhancing the table method, either creating and detailing requirements or applying different weights for these requirements, represented by liat equation(1).

\section{CONCLUSION}

DCIM is a center pillar that separates a sophisticated data center from a merely modern one. Instead of several DCIM solutions that are either contrary or with overlapping functionalities, Datacenter infrastructure managementaware equipment will be able to communicate and work collectively.

The Data centers became the core of Information Technology (IT) and now-a-days are top consumers of energy, comparable to big manufacture plants. Due to the significance of data for all activities, data center availability and immediate accessibility is the focal point. Automation systems devoted to datacenter accessibility, economy and management are emerging forces and will represent expressive part of all new data center installations. These automation systems, called DCIM, do not meet yet a standard or a common pattern.

A proposal is presented for a DCIM Evaluation Method, which is depending on a number of feature requirements, both for hardware and software. Five are the criteria projected to analyze: data, automation, management, interface and diagnosis. The process is dynamical and new evaluation requirements can be added to it anytime, as a means to improve it regularly.

Two current commercial platforms were analyzed in this paper and results were shown as a sample of the method possibilities. Future work will demonstrate a survey of commercial DCIM products, submitted to this Evaluation Method.

\section{REFERENCES}

1. Cabeamento estruturado para edifícios comerciais e data centers, ABNT NBR 14565/2011,2012.

2. Classification datacenter infrastructure tool Revision 3 by kevin brown dennis bouley

3. Data centre Infrastructure management by Dave cole

4. Achieving Data Centre energy effiency by jim witham

5. M. Faccioni Filho, "Plataformas online para gestão e monitoramento da infraestrutura de data centers," in IV Congresso RTI Data Centers 2012, São Paulo, SP,2012.

6. D. J. Cappuccio, “DCIM: Going Beyond IT,” Gartner Inc, Stamford, CT, USA, G00174769, March2010.

7. Data Center Site Infrastructure Tier Standard: Topology, Uptime Institute, LLC,2010.

8. Data Center Site Infrastructure Tier Standard: Operational Sustainability, Uptime Institute, LLC, 2010.

9. V. Avelar, "Guidance for Calculation of Efficiency (PUE) in Data Centers," Schneider Electric, Rueil Malmaison, France, White Paper 158 Rev 2,2011.

10. K. Broderick, "IDC MarketScape: Worldwide Datacenter Infrastructure Management (DCIM) 2011 Vendor Analysis," IDC, Framingham, MA USA, IDC\#232449 Volume 1, 2012.

11. Software Engineering Institute. CMMI (Capability Maturity Model Integration)

12. Available: http://www.sei.cmu.edu/cmmi/.
13. Data Center Design and Implementation Best Practices, ANSI/BICSI 002-2011,2011.

14. N. Nithiyanandam, K. Venkatesh, M. Rajesh, Transfer The Levels Of The Monitored Carbon, Nitrogen Gases From The Industries, International Journal of Recent Technology and Engineering, Volume-7 Issue-6S3 April, 2019.

15. Sivanesh Kumar, A., Brittoraj, S., Rajesh, M., Implementation of RFID with internet of things, Journal of Recent Technology and Engineering, Volume-7 Issue-6S3 April, 2019.

16. Rajesh, M., Sairam, R., Big data and health care system using mlearningJournal of Recent Technology and Engineering, Volume-7 Issue-6S3 April, 2019.

17. Rajesh, M., and J. M. Gnanasekar. "Path Observation Based Physical Routing Protocol for Wireless Ad Hoc Networks." Wireless Personal Communications 97.1 (2017): 1267-1289. 